\title{
Collagens and proteoglycans of the corneal extracellular matrix
}

Y.M. Michelacci

\author{
Correspondence \\ Y.M. Michelacci \\ Departamento de Bioquímica \\ EPM, UNIFESP \\ Rua Três de Maio, 100 \\ 04044-020 São Paulo, SP \\ Brasil \\ Fax: +55-11-5573-6407 \\ E-mail: yara.bioq@epm.br \\ Presented at SIMEC 2002 \\ (International Symposium \\ on Extracellular Matrix), \\ Angra dos Reis, RJ, Brazil, \\ October 7-10, 2002
}

Research supported by FAPESP,

CNPq, CAPES, and Sociedade Paulista

para o Desenvolvimento da Medicina

(SPDM).

Received December 13, 2002

Accepted January 27, 2003
Departamento de Bioquímica, Escola Paulista de Medicina,

Universidade Federal de São Paulo, São Paulo, SP, Brasil

\begin{abstract}
The cornea is a curved and transparent structure that provides the initial focusing of a light image into the eye. It consists of a central stroma that constitutes $90 \%$ of the corneal depth, covered anteriorly with epithelium and posteriorly with endothelium. Its transparency is the result of the regular spacing of collagen fibers with remarkably uniform diameter and interfibrillar space. Corneal collagen is composed of heterotypic fibrils consisting of type I and type $\mathrm{V}$ collagen molecules. The cornea also contains unusually high amounts of type VI collagen, which form microfibrillar structures, FACIT collagens (XII and XIV), and other nonfibrillar collagens (XIII and XVIII). FACIT collagens and other molecules, such as leucine-rich repeat proteoglycans, play important roles in modifying the structure and function of collagen fibrils. Proteoglycans are macromolecules composed of a protein core with covalently linked glycosaminoglycan side chains. Four leucine-rich repeat proteoglycans are present in the extracellular matrix of corneal stroma: decorin, lumican, mimecan and keratocan. The first is a dermatan sulfate proteoglycan, and the other three are keratan sulfate proteoglycans. Experimental evidence indicates that the keratan sulfate proteoglycans are involved in the regulation of collagen fibril diameter, and dermatan sulfate proteoglycan participates in the control of interfibrillar spacing and in the lamellar adhesion properties of corneal collagens. Heparan sulfate proteoglycans are minor components of the cornea, and are synthesized mainly by epithelial cells. The effect of injuries on proteoglycan synthesis is discussed.
\end{abstract}

\section{Cornea}

The cornea is a curved and transparent structure that, together with the sclera, composes the fibrous tunic of the eye. The fully developed cornea consists of a thick central portion known as the stroma, which constitutes about $90 \%$ of the corneal depth, covered anteriorly with stratified epithelium and posteriorly with endothelium. Both cell lay-
Key words

- Cornea

- Proteoglycan

- Glycosaminoglycan

- Collagen

- Dermatan sulfate

- Keratan sulfate 
relevant aspects of collagen biosynthesis have been recognized in recent years, we are still far from understanding the mechanisms underlying this uniformly oriented and perfectly calibrated synthesis and deposition of collagen fibers in the corneal matrix. Proteoglycans are thought to play an important role in this process. The present review deals with the most prominent macromolecules of the corneal extracellular matrix: collagen and proteoglycans.

\section{Collagen}

Collagen is the most abundant protein in vertebrates. It is a major component of the extracellular matrix and 26 different types of collagen have been described in vertebrates thus far $(1,2)$. Table 1 summarizes the families and types of collagen. The fibril-forming collagens are the most abundant and include

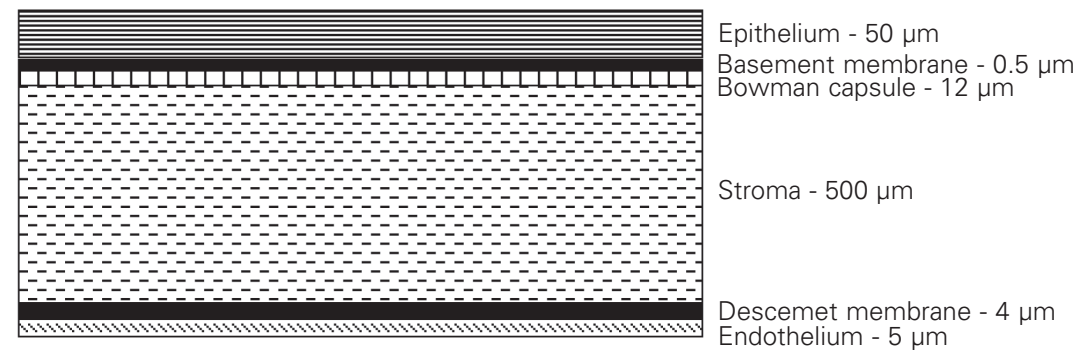

Figure 1. Schematic representation of human corneal layers. Stroma comprises about $90 \%$ of the corneal depth and is covered anteriorly with an epithelial layer and posteriorly with endothelium. Both layers are separated from the stroma by basement membranes. Only epithelial, stromal and endothelial layers are cellular.

Table 1. Types of collagens.

Collagen types

\begin{tabular}{|c|c|}
\hline Fibril forming & $I, I I, I I I, V, X I$ \\
\hline FACIT & IX, XII, XIV, XVI, XIX \\
\hline \multicolumn{2}{|l|}{ Nonfibrillar } \\
\hline Short chain & VIII, X \\
\hline Basement membrane & IV \\
\hline Anchoring fibrils & VII \\
\hline Microfibrillar & $\mathrm{VI}$ \\
\hline Other collagens & $X I I I, X V, X V I I, X V I I I, X X, X X I, X X I I, X X I I I, X X I V, X X V, X X V I$ \\
\hline
\end{tabular}

FACIT $=$ fibril-associated collagens with interrupted triple helices. types I, II, III, V, VI and XI (3). Other collagens, known as FACIT collagens (fibrilassociated collagens with interrupted triple helices), associate with the surface of collagen fibrils and modify their interactive properties $(4,5)$. In addition, nonfibrillar collagens such as types XIII, XVII and XXV have been reported to be localized on the cell surface $(6,7)$.

Despite the differences among collagens, all share a triple-helix structure composed of three polypeptides, known as $\alpha$ chains, consisting of Gly-X-Y repeats, where $X$ is any amino acid, and $Y$ is frequently proline or hydroxyproline. Each chain is a left-handed helix, and the three chains wind around each other in a right-handed superhelix.

\section{Collagen biosynthesis}

Collagen $\alpha$ chains are synthesized in the rough endoplasmic reticulum (RER) as long precursors named proo chains. For example, type I procollagen is a heterotrimer that contains two prool(I) chains encoded by the COL1A1 gene, and one proo2(I) chain encoded by the COL1A2 gene (Figure 2). The preproo chains contain different structural domains: a) a signal sequence of 20 amino acids that is cleaved during RER transit, $b$ ) the N-terminal propeptide, c) a short nontriple-helix domain that contains the site of proteolytic cleavage of $\mathrm{N}$-terminal peptides, d) the major triple-helix domain containing 1014 amino acid residues (each chain), characterized by the Gly-X-Y repeats, e) a 28residue telopeptide, and f) the final 220 residues that form a globular structure containing disulfide bonds (C-terminal propeptide).

A large number of enzymes are involved in the post-translation modifications of the $\alpha$ chains (Figure 3). Some of these modifications, such as hydroxylation of proline to form 3- or 4-hydroxyproline, hydroxylation of lysine to hydroxylysine, and glycosylation of hydroxylysine, occur during the elongation of the peptide in the RER, and are 

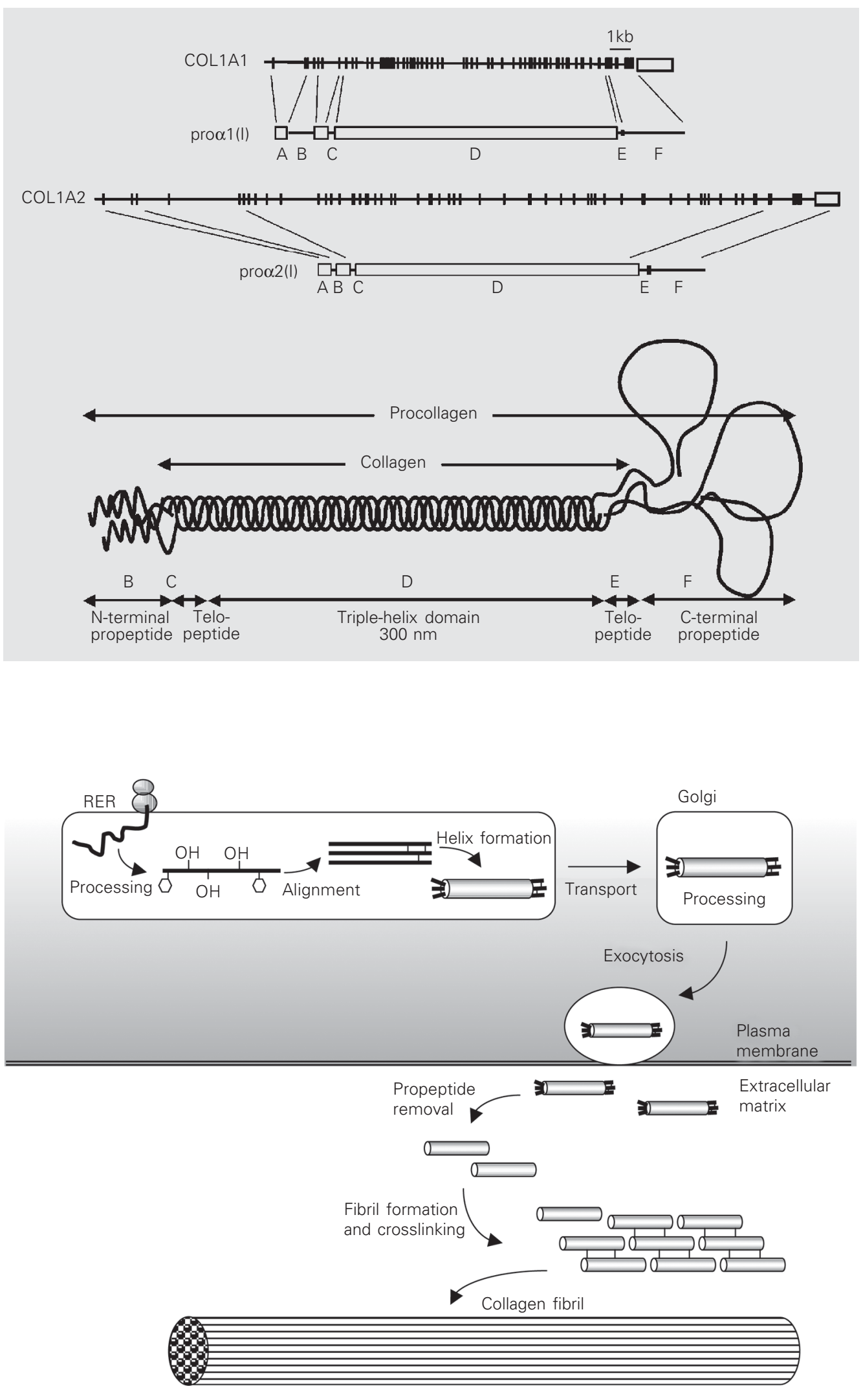

Figure 2. Genes that encode type I preprocollagen $\alpha$ chains (top) and domain structure of procollagen molecule (bottom). $A$, Signal sequence that is cleaved during rough endoplasmic reticulum transit; $B, \mathrm{~N}$-terminal propeptide; $C$, short nontriple-helix domain that contains the site of proteolytic cleavage of $\mathrm{N}$-terminal peptides; $D$, major triple-helix domain, characterized by the Gly-X-Y repeats; $E$, telopeptide; $F, \mathrm{~N}$-terminal propeptides that contain disulfide bonds.

Figure 3. Biosynthesis of fibrillar collagens. Modifications of the procollagen peptide in the rough endoplasmic reticulum (RER) include hydroxylation, glycosylation, and disulfide bond formation. Interchain disulfide bonds between the C-terminal peptides align the three chains and initiate formation of the triple helix. The process propagates towards the $\mathrm{N}$-terminus of the molecule end. N-linked oligosaccharides are transferred to the propeptides in the RER and processed in the Golgi apparatus. Upon secretion, propeptides are cleaved, allowing lateral alignment and crosslinking. See text for details. 
followed by formation of the triple helix Prolyl 4-hydroxylase, located either in the lumen or on the inner surface of the RER membrane, is a tetramer containing two $\alpha$ subunits $(64 \mathrm{kDa})$ and two $\beta$ subunits (60 $\mathrm{kDa}$ ) which are products of different genes (8). The $\beta$ subunits contain the $C$-terminal amino acid sequence Lys-Asp-Glu-Leu (KDEL) that confers the ability to reside permanently in the RER. Prolyl 3-hydroxylase has a molecular mass of $160 \mathrm{kDa}$, and details about the structure of this enzyme have been recently reported (9). Lysyl hydroxylase is a homodimer, the monomer of which has a molecular mass of $85 \mathrm{kDa}(10$, see Ref. 11 for a review on these enzymes).

The extent of post-translation modification of prolyl and lysyl residues in the diverse collagen types differs substantially. For example, the relative extent of lysyl hydroxylation in type IV collagen is very high (more than $90 \%$ of the residues at position $\mathrm{Y}$ are hydroxylated), while in types I and III only $15 \%$ are hydroxylated. The three collagen hydroxylases require $\mathrm{Fe}^{2+}$, 2-oxoglutarate, molecular oxygen $\left(\mathrm{O}_{2}\right)$ and ascorbate as co-factors.

The hydroxylation of proline at the 4 position is essential to provide thermal stability to the triple helix. Hydroxylation of lysyl residues provides a substrate for both glycosylation and formation of stable crosslinks, important to the tissue tensile strength (12).

The glycosylation of hydroxylysyl residues in collagen requires two enzymes: hydroxylysyl galactosyltransferase and galactosylhydroxylysyl glucosyltransferase (13). The former adds a galactose residue to the 5-OH of hydroxylysine, and the latter transfers glucose to the galactose residue. The distribution of monosaccharides and disaccharides is influenced by the collagen type and is carried out only on non-triplehelix substrates.

Furthermore, asparagine-linked oligosaccharides may be present in the propeptide extensions (14). These oligosaccharides are synthesized on a dolichol lipid intermediate in the RER membrane and transferred intact to the pro $\alpha$ chain (15). There is initial cleavage of the oligosaccharide while the chain is still in the RER, and further processing in the Golgi apparatus (16).

After synthesis is completed, the globular domains of the proo chains fold and are stabilized by disulfide bonds. The triplehelix formation begins at the $\mathrm{C}$-terminal end of the molecule and is propagated towards the N-terminal end. The propagation of the triple-helix structure requires all peptide bonds involving the prolyl residues to be in the trans form, a configuration possibly produced by the enzyme peptidyl prolyl cistrans isomerase (17).

Procollagen molecules are translocated to the Golgi apparatus and then packaged in secretory vesicles that fuse with the cell membrane and release their contents into the extracellular environment. In the Golgi apparatus, N-linked oligosaccharides are trimmed and synthesis of a high-mannose structure occurs. In addition, phosphorylation of certain serine residues and sulfation of some tyrosine residues occur in some collagens.

Once outside the cell, procollagens undergo proteolytic conversion to collagen, form fibrils, interact with other proteins, are stabilized by intermolecular crosslinks, and are eventually degraded. Propeptides are removed by two enzymes: procollagen N-proteinase and procollagen C-proteinase (18). Once cleaved, collagen molecules rapidly aggregate into ordered structures. Fibril formation is a nonenzymatic process and the nature of the interactions that govern fibril diameter is not completely understood. Interactions with other types of collagen or with proteoglycans are thought to control the rate of fibrillogenesis and the fibril diameter (19).

Collagen molecules in fibrillar array become substrate for the enzyme lysyl oxidase 
that oxidatively deaminates certain lysyl and hydroxylysyl residues (20), forming reactive aldehydes that condense with lysyl or hydroxylysyl residues in adjacent molecules to form divalent crosslinks (Figure 3). Lysyl oxidase is a monomeric enzyme of $32 \mathrm{kDa}$ (21) and requires pyridoxyl and copper as co-factors.

\section{Corneal collagens}

Table 2 summarizes the different collagen types described in vertebrate corneas, as well as the relative amounts of the most abundant ones. Normal human corneal stroma is rich in type I collagen, but also contains relatively large amounts of type $\mathrm{V}$ (22) and type VI collagens (23). Type III is present at low proportions but increases during wound healing, inflammation and several pathological conditions.

Corneal collagen fibrils are composed of type I collagen molecules incorporated together with those of type $\mathrm{V}$ collagen into heterotypic fibrils (24). Type VI collagen forms microfibrillar structures by lateral aggregation, and types XII and XIV collagens are FACIT collagens (25). By analogy with type IX collagen in cartilage, the $\mathrm{N}$-terminal domains of type XII and XIV collagens might be expected to protrude from the surface of corneal collagen fibrils where they would be able to interact with proteins in the corneal stroma.

Collagen XIII, a nonfibrillar collagen that is bound to the cell membrane through a single transmembrane domain, is widely expressed in the human eye and has been found in the posterior two thirds of the corneal stroma (7).

Collagen XVIII is a proteoglycan localized in the basement membrane of epithelia and vascular endothelium. Collagen XVIII is the only currently known collagen that carries heparan sulfate glycosaminoglycan side chains (26). Collagen XVIII gained public attention when it was discovered that part of its C-terminal non-triple-helix domain is endostatin, a potent inhibitor of angiogenesis and tumor growth released by cleavage of an Ala-His peptide bound (27). The processing of collagen XVIII to endostatin may represent a local control mechanism for the regulation of angiogenesis. In the cornea, collagen XVIII was immunolocalized to the human corneal epithelium, epithelial basement membrane, and Descemet membrane (28), and lack of collagen XVIII was shown to cause eye abnormalities (29).

\section{Proteoglycans}

In addition to FACIT collagens, other molecules have important roles in modifying the structure and function of collagen fibrils. These include some leucine-rich repeat proteoglycans (for a review, see Ref. 30).

Proteoglycans are macromolecules composed of a protein core with covalently linked glycosaminoglycan side chains (31). Studies of the glycosaminoglycans from bovine (32), rabbit (33), chicken (34), monkey (35) and human (36) corneas have shown that dermatan sulfate and keratan sulfate are the predominant components, with smaller amounts of heparan sulfate. The keratan sulfate chain

\begin{tabular}{llr} 
Table 2. Corneal collagens. & \% of total \\
\hline Type & Localization & 75 \\
\hline I & Stroma & \\
II & Developing stroma (epithelium) \\
III & Inflammation, wound healing \\
IV & Basement membranes \\
V & Stroma \\
VI & Stroma \\
VII & Basement membrane \\
& $\quad$ (epithelium) \\
VIII & Basement membrane \\
& $\quad$ (Descemet) \\
IX & Developing stroma (epithelium) \\
XII & Stroma (endothelial origin) \\
XIII & Stroma (posterior two thirds) \\
XIV & Stroma \\
XVII & Developing stroma (hemi- \\
& desmosomes) \\
XVIII & Basement membrane (epithelium) \\
&
\end{tabular}


is attached to the proteoglycan core protein by an $\mathrm{N}$-acetylglucosamine of a mannosecontaining linkage oligosaccharide, which is $N$-linked to an asparagine residue of the protein (37). The linkage oligosaccharide is similar to biantennary complex-type $N$-linked oligosaccharides found in glycoproteins, with the keratan sulfate extending one branch and sialic acid terminating the second branch (38) (Figure 4).

In 1992, Blochberger et al. (39) isolated a cDNA clone encoding the core protein of a chick corneal keratan sulfate proteoglycan, which they named lumican. Three keratan sulfate proteoglycan core proteins with different primary structures, named 37A, 37B and 25, were isolated from bovine cornea. These proteins were also cloned, and 37B was identified as the bovine lumican (40), with $68 \%$ identity to chicken lumican. The keratan sulfate proteoglycans containing the $37 \mathrm{~A}$ and the 25 cores were named keratocan and mimecan (or osteoglycin), respectively $(41,42)$ (Figure 5). It seems that although expressed in other tissues, only in the cornea are lumican, keratocan, and mimecan glycosylated with sulfated keratan sulfate chains.

The corneal proteoglycans are thought to play a role in collagen fibrillogenesis and matrix assembly. Morphological studies have shown both dermatan sulfate and keratan sulfate proteoglycans to be associated with specific bands of corneal collagen fibrils $(43,44)$, suggesting that proteoglycan-collagen interactions may play a role in corneal fibril assembly, matrix organization and ultimately corneal transparency.

The crucial role of lumican in the regulation of collagen assembly into fibrils has been established by studies on mice homozygous for a null mutation in lumican. These animals presented skin laxity and fragility and bilateral corneal opacity (45). In addition, it was shown that people who have mutations in KERA, the gene encoding keratocan, have cornea plana (46), in which the forward convex curvature is flattened leading to a decrease in refraction. Furthermore, it was recently shown that mimecan-deficient mice have thicker collagen fibrils in
Figure 4. Structure of corneal matrix glycosaminoglycans showing the polysaccharide-protein linkage region and the main disaccharide units. Gal, galactose; Fuc, fucose; Man, mannose; Xyl, xylose; GluA, ß-D-glucuronic acid; IduA, $\alpha$-L-iduronic acid; GalNAc, N-acetylgalactosamine; GalNAc4S, N-acetylgalactosamine 4-sulfate; GlcNAc, Nacetylglucosamine; GlcNAc6S, $\mathrm{N}$-acetylglucosamine 6-sulfate; Asp, asparagine; Ser, serine.

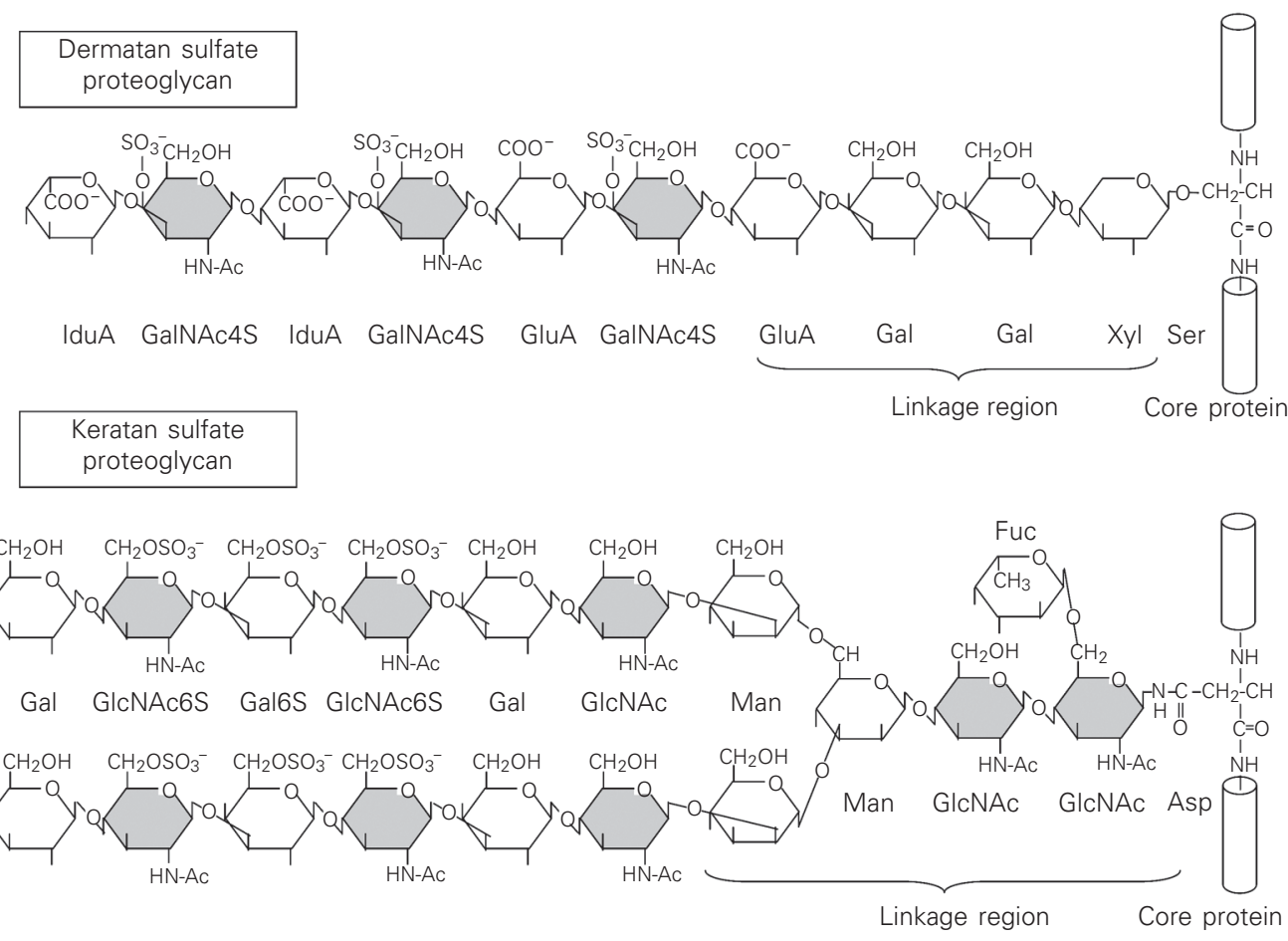


both corneal and skin preparations (47).

The non-glycosylated core protein of lumican is as effective as the intact proteoglycan in inhibiting fibrillogenesis in vitro (48). Nevertheless, studies on chick embryo corneal development suggest that the glycosylation is very important for the corneal transparency process (49). Lumican with nonsulfated keratan sulfate side chains was detected as early as on day 7 of embryonic development of chicken, but sulfated glycosaminoglycan side chains were detected only on day 15 , when transparency starts to increase. Also in embryonic mouse, sulfated keratan sulfate proteoglycans appeared only after the eyes opened (50). It was also shown that alterations in sulfation or in the fine structure of the keratan sulfate chains occur during chick corneal maturation for these three keratan sulfate proteoglycans (51). These findings suggest that the structure of glycosaminoglycan chains in keratan sulfate proteoglycans may be important for the development of corneal transparency, possibly leading to organization and optimal hydration of the corneal tissue.
The fourth leucine-rich repeat proteoglycan of the cornea is a dermatan sulfate proteoglycan (Figure 4). The corneal dermatan sulfate chains are linked to a $40-\mathrm{kDa}$ protein, forming a proteoglycan of 100-150 kDa containing one or two glycosaminoglycan side chains (52). The core protein was originally characterized by its antigenic properties as decorin (Figure 5), a proteoglycan present in most connective tissues and structurally related to other small interstitial proteoglycans. Later, $\mathrm{Li}$ et al. (53) confirmed this identity by cloning and sequencing a cDNA encoding the core protein of chick corneal dermatan sulfate proteoglycan.

Hahn and Birk (54) studied the effects of B-D-xyloside on the fibril organization of avian cornea. This compound interferes with xylose-mediated $O$-linked proteoglycan synthesis and thus disturbs dermatan sulfate proteoglycan synthesis, but does not modify the keratan sulfate proteoglycan formation. The collagen fibril diameters were unaltered, but a disruption in lamellar organization occurred, suggesting that dermatan sulfate proteoglycans are not involved in the regulation

$\begin{array}{ll}\text { Proteoglycan } & \text { Gignal peptide } \\ \text { Class I } & \text { Decorin } \\ \text { Biglycan } & \text { (number of chains) }\end{array}$

Figure 5. Domain structure of decorin, a prototype member of the leucine-rich repeat proteoglycan family, and classification of the leucine-rich repeat proteoglycans based on amino acid sequence homology. Roman numerals indicate decorin domains. In addition to the glycosaminoglycan chain (dermatan sulfate or chondroitin sulfate), N-linked oligosaccharides are also present. 
of collagen fibril diameter, but are important to fibril-fibril spacing and lamellar cohesiveness. In corneal explants from embryonic chicken, an increased synthesis of keratan sulfate proteoglycan and a decreased synthesis of dermatan sulfate proteoglycan coincide with the onset of tissue transparency, again suggesting a correlation between proteoglycan composition and corneal transparency (55).

Although several groups have studied the glycosaminoglycan composition of bovine, rabbit, chicken, and monkey cornea, as well as the biosynthesis of proteoglycans by rabbit and embryonic chicken corneal explants (56), only a few reports concerning human corneal glycosaminoglycans have appeared (57). The glycosaminoglycan (36) and proteoglycan (58) composition of human cornea, as well as the glycosaminoglycans synthesized by human cornea explants under tissue culture conditions have been recently described.

Corneal explants submitted to tissue culture conditions for three days presented the same glycosaminoglycan composition as the fresh cornea (dermatan sulfate and keratan sulfate, about $50 \%$ each, $1.5 \mathrm{mg} / \mathrm{g}$ tissue wet weight). The glycosaminoglycan synthesis was maintained in vitro for weeks under these tissue culture conditions, as indicated by the metabolic incorporation of $\left[{ }^{35} \mathrm{~S}\right]$-sulfate. Nevertheless, during a 24-h incubation the corneal explants synthesized glycosaminoglycans which varied in relative proportions from those present in intact corneas. The labeling of dermatan sulfate was higher than that of keratan sulfate. Furthermore, $\left[{ }^{35} \mathrm{~S}\right]$-heparan sulfate also appeared, suggesting a higher synthesis rate for dermatan sulfate and heparan sulfate.

The main glycosaminoglycan synthesized by epithelial cells isolated from human cornea is heparan sulfate. Conversely, stromal cells synthesize mainly dermatan sulfate and keratan sulfate when both epithelial and endothelial cell layers are removed (36).

The quantitative and qualitative patterns of proteoglycan synthesis, and possibly of collagen fibril deposition, change when the cornea is submitted to injuries, such as epithelial debridement $(36,58)$ and laser refractive surgery (59), suggesting that the keratocyte program for synthesis and deposition of extracellular matrix, which was followed during normal development leading to corneal transparency, is hardly recovered after injury.

Thus, studies to determine the structure of corneal fibrils and to identify in three dimensions the binding of FACIT collagens, leucine-rich repeat proteoglycans, and other macromolecules, are directly relevant to understanding the assembly of the corneal stroma in health and disease.

\section{References}

1. Myllyharju J \& Kivirikko KI (2001). Collagens and collagen related diseases. Annals of Medicine, 33: 7-21.

2. Sato K, Yomogida K, Wada T, Yorihuzi T, Nishimune Y, Hosokawa N \& Nagata K (2002). Type XXVI collagen, a new member of the collagen family, is specifically expressed in the testis and ovary. Journal of Biological Chemistry, 277: 37678-37684.

3. Kadler KE, Holmes DF, Trotter JA \& Chapman JA (1996). Collagen fibril formation. Biochemical Journal, 316: 1-11.

4. Koch M, Foley JE, Hahn R, Zhou P, Burgeson RE, Gerecke DR \& Gordon MK (2001). Alpha $1(X X)$ collagen, a new member of the collagen subfamily, fibril-associated collagens with interrupted triple helices. Journal of Biological Chemistry, 276: 23120-23126.

5. Shaw LM \& Olsen BR (1991). FACIT collagens: diverse molecular bridges in extracellular matrices. Trends in Biochemical Sciences, 16: 191-194.

6. Snellman $A, T u H$, Vaisanen $T$, Kvist $A P$, Huhtala $P$ \& Pihlajaniemi $T$ (2000). A short sequence in the $\mathrm{N}$-terminal region is required for the trimerization of type XIII collagen and is conserved in other collagenous transmembrane proteins. EMBO Journal, 19: 5051-5059.

7. Sandberg-Lall M, Hagg PO, Wahlstrom I \& Pihlajaniemi T (2000). Type XIII collagen is widely expressed in the adult and developing human eye and accentuated in the ciliary muscle, the optic nerve and the neural retina. Experimental Eye Research, 70: 401-410.

8. Pihlajaniemi T, Helaakoski T, Tasanen K, Myllyla R, Huhtala ML, Koivu J \& Kivirikko KI (1987). Molecular cloning of the beta-subunit of human prolyl 4-hydroxylase. This subunit and protein disulphide 
isomerase are products of the same gene. EMBO Journal, 6: 643649.

9. Clifton IJ, Hsueh LC, Baldwin JE, Harlos K \& Schofield CJ (2001). Structure of proline 3-hydroxylase. Evolution of the family of 2oxoglutarate dependent oxygenases. European Journal of Biochemistry, 268: 6625-6636.

10. Turpeenniemi-Hujanen TM, Puistola U \& Kivirikko KI (1980). Isolation of lysyl hydroxylase, an enzyme of collagen synthesis, from chick embryos as a homogeneous protein. Biochemical Journal, 189: 247253.

11. Kivirikko KI \& Pihlajaniemi T (1998). Collagen hydroxylases and the protein disulfide isomerase subunit of prolyl 4-hydroxylases. Advances in Enzymology and Related Areas of Molecular Biology, 72: 325-398.

12. Eyre DR, Paz MA \& Gallop PM (1984). Cross-linking in collagen and elastin. Annual Review of Biochemistry, 53: 717-748.

13. Kivirikko KI \& Myllyla R (1979). Collagen glycosyltransferases. International Review of Connective Tissue Research, 8: 23-72.

14. Anttinen H, Oikarinen A, Ryhanen L \& Kivirikko KI (1978). Evidence for the transfer of mannose to the extension peptides of procollagen within the cisternae of the rough endoplasmic reticulum. FEBS Letters, 87: 222-226.

15. Housley TJ, Rowland FN, Ledger PW, Kaplan J \& Tanzer ML (1980). Effects of tunicamycin on the biosynthesis of procollagen by human fibroblasts. Journal of Biological Chemistry, 255: 121-128.

16. Clark CC (1982). Asparagine-linked glycosides. Methods in Enzymology, 82 (Part A): 346-360.

17. Bächinger HP, Bruckner P, Timpl R \& Engel J (1978). The role of cistrans isomerization of peptide bonds in the coil leads to and comes from triple helix conversion of collagen. European Journal of Biochemistry, 90: 605-613.

18. Tuderman L \& Prockop DJ (1982). Procollagen N-proteinase. Properties of the enzyme purified from chick embryo tendons. European Journal of Biochemistry, 125: 545-549.

19. Vogel KG, Paulsson M \& Heinegard D (1984). Specific inhibition of type I and type II collagen fibrillogenesis by the small proteoglycan of tendon. Biochemical Journal, 223: 587-597.

20. Siegel RC (1979). Lysyl oxidase. International Review of Connective Tissue Research, 8: 73-118.

21. Bank RA \& van Hinsbergh VW (2002). Lysyl oxidase: new looks on LOX. Arteriosclerosis, Thrombosis, and Vascular Biology, 22: 13651366.

22. Lee RE \& Davison PF (1984). The collagens of the developing bovine cornea. Experimental Eye Research, 39: 639-652.

23. Zimmermann DR, Trueb B, Winterhalter KH, Witmer R \& Fischer RW (1986). Type VI collagen is a major component of the human cornea. FEBS Letters, 197: 55-58.

24. Birk DE, Fitch JM, Babiarz JP \& Linsenmayer TF (1988). Collagen type $\mathrm{I}$ and type $\mathrm{V}$ are present in the same fibril in the avian corneal stroma. Journal of Cell Biology, 106: 999-1008.

25. Gordon MK, Foley JW, Linsenmayer TF \& Fitch JM (1996). Temporal expression of types XII and XIV collagen mRNA and protein during avian corneal development. Developmental Dynamics, 206: 49-58.

26. Dong S, Cole GJ \& Halfter W (2003). Expression of collagen XVIII and localization of its glycosaminoglycan attachment sites. Journal of Biological Chemistry, 278: 1700-1707.

27. Sasaki T, Fukai N, Mann K, Gohring W, Olsen BR \& Timpl R (1998). Structure, function and tissue forms of the C-terminal globular domain of collagen XVIII containing the angiogenesis inhibitor endostatin. EMBO Journal, 17: 4249-4256.

28. Lin HC, Chang JH, Jain S, Gabison EE, Kure T, Kato T, Fukai N \& Azar
DT (2001). Matrilysin cleavage of corneal collagen type XVIII NC1 domain and generation of a $28-\mathrm{kDa}$ fragment. Investigative Ophthalmology and Visual Science, 42: 2517-2524.

29. Fukai N, Eklund L, Marneros AG et al. (2002). Lack of collagen XVIII/ endostatin results in eye abnormalities. EMBO Journal, 21: 15351544

30. lozzo RV (1999). The biology of the small leucine-rich proteoglycans. Functional network of interactive proteins. Journal of Biological Chemistry, 274: 18843-18846.

31. Hassell JR, Kimura JH \& Hascall VC (1986). Proteoglycan core protein families. Annual Review of Biochemistry, 55: 539-567.

32. Axelsson I \& Heinegard D (1978). Characterization of the keratan sulfate proteoglycans from bovine corneal stroma. Biochemical Journal, 169: 517-530.

33. Gregory JD, Coster L \& Damle SP (1982). Proteoglycans of rabbit corneal stroma. Isolation and partial characterization. Journal of Biological Chemistry, 257: 6965-6970.

34. Funderburgh JL, Caterson B \& Conrad GW (1986). Keratan sulfate proteoglycan during embryonic development of the chicken cornea. Developmental Biology, 116: 267-277.

35. Hassell JR, Newsome DA \& Hascall VC (1979). Characterization and biosynthesis of proteoglycans of corneal stroma from Rhesus monkey. Journal of Biological Chemistry, 254: 12346-12354.

36. Soriano ES, Campos MS \& Michelacci YM (2000). Effect of epithelial debridement on glycosaminoglycan synthesis by human corneal explants. Clinica Chimica Acta, 295: 41-62.

37. Stein K, Keller R, Stuhlsatz HW, Greiling H, Ohst E, Muller E \& Scharf $H-D$ (1982). Structure of the linkage region between polysaccharide chain and core protein in bovine corneal proteo-keratan sulfate. Hoppe-Seyler's Zeitschrift für Physiologische Chemie, 363: 825-833.

38. Nilsson B, Nakazawa K, Hassell JR, Newsome DA \& Hascall VC (1983). Structure of oligosaccharides and their linkage region between keratan sulfate and the core protein of proteoglycans from monkey cornea. Journal of Biological Chemistry, 258: 6056-6063.

39. Blochberger TC, Vergnes J-P, Hempel J \& Hassell JR (1992). cDNA to chick lumican (corneal keratan sulfate proteoglycan) reveals homology to the small interstitial proteoglycan gene family and expression in muscle and intestine. Journal of Biological Chemistry, 267: 347-352.

40. Funderburgh JL, Funderburgh ML, Brown SJ, Vergnes J-P, Hassell JR, Mann MM \& Conrad GW (1993). Sequence and structural implications of a bovine corneal keratan sulfate proteoglycan core protein. Protein 37B represents bovine lumican and proteins 37A and 25 are unique. Journal of Biological Chemistry, 268: 11874-11880.

41. Corpus LM, Funderburgh JL, Funderburgh ML, Bottomley GS, Prakash S \& Conrad GW (1996). Molecular cloning and tissue distribution of keratocan. Bovine corneal keratan sulfate proteoglycan 37A. Journal of Biological Chemistry, 271: 9759-9763.

42. Funderburgh JL, Corpus LM, Roth MR, Funderburgh ML, Tasheva ES \& Conrad GW (1997). Mimecan, the 25-kDa corneal keratan sulfate proteoglycan, is a product of the gene producing osteoglycin. Journal of Biological Chemistry, 272: 28089-28095.

43. Scott JE \& Haigh M (1985). "Small"-proteoglycan:collagen interactions: keratan sulphate proteoglycan associates with rabbit corneal collagen fibrils at the " $a$ " and "c" bands. Bioscience Reports, 5: 765-774.

44. Scott JE \& Haigh M (1988). Identification of specific binding sites for keratan sulphate proteoglycans and chondroitin-dermatan sulphate proteoglycans on collagen fibrils in cornea by the use of cupromeronic blue in critical-electrolyte-concentration techniques. Biochemical Journal, 253: 607-610. 
45. Chakravarti S, Magnuson T, Lass JH, Jepson KJ, LaMantia C \& Carroll H (1998). Corneal opacity in lumican-null mice: defects in collagen fibril structure and packing in the posterior stroma. Journal of Cell Biology, 141: 1277-1286.

46. Pellegata NS, Dieguez-Lucena JL, Joensuu T, Lau S, Montgomery KT, Krahe R, Kivela $T$, Kucherlapati R, Forsius $H$ \& de la Chapelle A (2000). Mutations in KERA, encoding keratocan, cause cornea plana. Nature Genetics, 25: 91-95

47. Tasheva ES, Koester A, Paulsen AQ, Garrett AS, Boyle DL, Davidson HJ, Song M, Fox N \& Conrad GW (2002). Mimecan/osteoglycindeficient mice have collagen fibril abnormalities. Molecular Vision, 8: 407-415.

48. Rada JA, Cornuet PK \& Hassell JR (1993). Regulation of corneal collagen fibrillogenesis in vitro by corneal proteoglycan (lumican and decorin) core proteins. Experimental Eye Research, 56: 635-648.

49. Cornuet PK, Blochberger TC \& Hassell JR (1994). Molecular polymorphism of lumican during corneal development. Investigative Ophthalmology and Visual Science, 35: 870-877.

50. Ying S, Shiraishi A, Kao CW, Converse RL, Funderburgh JL, Swiergiel J, Roth MR, Conrad GW \& Kao WW (1997). Characterization and expression of the mouse lumican gene. Journal of Biological Chemistry, 272: 30306-30313.

51. Dunlevy JR, Beales MP, Berryhill BL, Cornuet PK \& Hassell JR (2000). Expression of the keratan sulfate proteoglycans lumican, keratocan and osteoglycin/mimecan during chick corneal development. Experimental Eye Research, 70: 349-362.

52. Midura RJ \& Hascall VC (1989). Analysis of the proteoglycans synthesized by corneal explants from embryonic chicken. II. Structural characterization of the keratan sulfate and dermatan sulfate proteo- glycans from corneal stroma. Journal of Biological Chemistry, 264: 1423-1430

53. Li W, Vergnes J-P, Cornuet PK \& Hassell JR (1992). cDNA clone to chick corneal chondroitin/dermatan sulfate proteoglycans reveals identity to decorin. Archives of Biochemistry and Biophysics, 296: 190-197.

54. Hahn RA \& Birk DE (1992). ß-D-xyloside alters dermatan sulfate synthesis and the organization of the developing avian corneal stroma. Development, 115: 383-393.

55. Nakazawa K, Suzuki S, Wada K \& Nakazawa K (1995). Proteoglycan synthesis by corneal explants from developing embryonic chicken. Journal of Biochemistry, 117: 707-718.

56. Midura RJ, Toledo OMS, Yanagishita M \& Hascall VC (1989). Analysis of the proteoglycans synthesized by corneal explants from embryonic chicken. I. Characterization of the culture system with emphasis on stromal proteoglycan biosynthesis. Journal of Biological Chemistry, 264: 1414-1422.

57. Tai GH, Nisduszynski IA, Fullwood NJ \& Huckerby TN (1997). Human corneal keratan sulfates. Journal of Biological Chemistry, 272: $28227-$ 28231.

58. Soriano ES, Campos MS, Aguiar JA \& Michelacci YM (2001). Effect of epithelial debridement on human cornea proteoglycans. Brazilian Journal of Medical and Biological Research, 34: 325-331.

59. Martins SAR, Berto AGA, Aguiar JAK, Soriano ES \& Michelacci YM (2002). Proteoglycan synthesis after refractive surgery comparing two techniques: laser in situ keratomileusis (LASIK) and photorefractive surgery (PRK). Presented at SIMEC 2002 - International Symposium on Extracellular Matrix, Angra dos Reis, RJ, Brazil, October 710, 2002, E-11 (Abstract). 